\title{
On the Computation of All Imaginary Quadratic Fields of Class Number One
}

\author{
By Juergen M. Cherubini and Rolf V. Wallisser
}

\begin{abstract}
Let $d$ be the discriminant of an imaginary quadratic field with class number one. If $d \leqslant-10^{4}$ it is easy to show, using an idea from Stark, that $h(12 d) \leqslant 2 \sqrt{|d|}, h(24 d) \leqslant 2 \sqrt{|d|}$ and $|h(24 d) \ln (5+2 \sqrt{6})-2 h(12 d) \ln (2+\sqrt{3})|<50 \exp (-\pi / 24 \cdot \sqrt{|d|})$. This linear form is estimated for large $|d|$ from below with the aid of the quantitative version of Schneider's $\alpha^{\beta}$-theorem by Mignotte and Waldschmidt. In the "medium large" region $2 \cdot 10^{4} \leqslant|d| \leqslant 10^{34}$ it is shown by computing the beginning of the continued fraction of $\ln (5+2 \sqrt{6}) / \ln (2+\sqrt{3})$ that the above relations cannot hold.
\end{abstract}

The well-known conjecture of Gauss [6] which says that there exist only nine imaginary quadratic fields with a unique factorization was verified by Heegner [9] in 1952. But mathematicians of that time thought the proof to be incomplete, and only when Stark [14] in 1967 gave his solution of the problem, was it shown that Heegner was quite correct [13]. While these proofs were based on the theory of modular functions, Baker [1], in 1967, showed that the conjecture of Gauss could also be solved, essentially by using his estimations of linear forms in logarithms of algebraic numbers. Then Bundschuh and Hock [2] gave a proof using Baker's idea, and shortly thereafter, Fel'dman and Chudakov [5] proved the theorem by using a method outlined by Gel'fond [7] already in 1939.

We provide here a further proof based on Mignotte and Waldschmidt's [11] quantitative version of Schneider's solution of Hilbert's seventh problem. The difference of the proofs using transcendental number theory is the following: Bundschuh and Hock used a linear form with integer coefficients in three logarithms of algebraic numbers. They showed that if there exists an imaginary quadratic field with class number one, the absolute value of the discriminant $d$ is bounded from above by $\exp \left(1.6 * 10^{5}\right)$. Fel'dman and Chudakov used a linear form in two logarithms of algebraic numbers with algebraic coefficients and got the upper bound $|d|<10^{40}$. To finish the proof, both used the strong bound of Stark [12], who showed that for the discriminant $d$ of a tenth imaginary quadratic field of class number one, $|d|>\exp \left(2.2 * 10^{7}\right)$ must hold. Here we also use a linear form in two logarithms of algebraic numbers, but with integer coefficients (see Stark [15, Section 9]). We estimate this linear form with the help of a theorem of Mignotte and Waldschmidt and find an upper bound $|d|<10^{34}$. Now, since our linear form has integer coefficients and the upper bound of the discriminant is "small", we can

Received May 5, 1986: revised November 12, 1986.

1980 Mathematics Subject Classification (1985 Revision). Primary 11J99, 11E41, 11 E45. 
finish the proof using continued fractions. So we avoid Stark's strong lower bound and get a proof which is more self-contained.

The authors are indebted to the referee for mentioning the work of Gross and Zagier [8] giving an effective lower bound for the class numbers of all imaginary quadratic fields. However, it seems that in case of class number one the algorithm is not better than ours because a "medium large" region remains which has to be handled by other methods. In this paper we wanted to show that today's lower bounds of linear forms in logarithms yield an effective algorithm to prove the conjecture of Gauss already on a personal computer.

1. The Connection of Gauss' Conjecture with the Estimation of a Linear Form in Two Logarithms. Let $d$ be the discriminant of the quadratic field $K=\mathbf{Q}(\sqrt{d})$, $\chi_{d}(n)$ the primitive real character $(d / n)$ and $L\left(s, \chi_{d}\right)$ the Dirichlet $L$-series

$$
L\left(s, \chi_{d}\right)=\sum_{n=1}^{\infty} \chi_{d}(n) n^{-s}, \quad \operatorname{Re} s>1 .
$$

Dirichlet showed the following relation between the class number $h(d)$ and $L\left(1, \chi_{d}\right)$ :

$$
\begin{gathered}
h(d)=\frac{1}{\kappa} L\left(1, \chi_{d}\right), \\
\kappa= \begin{cases}\frac{2 \pi}{w \sqrt{|d|}} & \text { if } d<0, \\
\frac{\ln \varepsilon}{\sqrt{|d|}} & \text { if } d>0\end{cases}
\end{gathered}
$$

( $w$ denotes the order of the group of units, $\varepsilon$ is the fundamental unit). If we assume $d<-4, h(d)=1$, and if $Q(x, y)$ is a positive-definite quadratic form with integral coefficients and discriminant $d, k$ the discriminant of a real quadratic field with $(k, d)=1$, and $L\left(s, \chi_{k}, Q\right)$ the Epstein zeta function

$$
L\left(s, \chi_{k}, Q\right)=2^{-1} \sum_{x, y \neq 0,0} \chi_{k}(Q(x, y)) Q(x, y)^{-s},
$$

then one has the relation

$$
L\left(s, \chi_{k}\right) L\left(s, \chi_{k d}\right)=L\left(s, \chi_{k}, Q\right) \text { for } \operatorname{Re} s>1 .
$$

From (1), (2), and (3) one gets (see Stark [15]), if $k$ is not the power of a prime number and $\varepsilon$ denotes the fundamental unit of $\mathbf{Q}(\sqrt{k})$,

$$
\begin{aligned}
& h(k) h(k d) \ln \varepsilon \\
& =\frac{\pi}{6} k \sqrt{|d|} \prod_{p \mid n}\left(1-p^{-2}\right) \\
& \quad+4 \sum_{n=1}^{\infty} e^{-\pi n \sqrt{|d|} / h} \sum_{\substack{y \mid n \\
y>0}} \frac{1}{y} \sum_{j=1}^{h} \chi_{k}(Q(j, y)) \exp \left(\frac{2 \pi i j n}{k y}+\frac{\pi j n}{k}\right) .
\end{aligned}
$$

With the aid of elementary considerations (see, e.g., Heilbronn and Linfoot [10]) one can show that the only imaginary quadratic fields $\mathbf{Q}(\sqrt{d})$ with class number one and even discriminant $d$ are $d=-4,-8$ and that for all other such fields, $|d|$ has to be a 
prime with $|d| \equiv 3(\bmod 4)$. It is easy to show that $h(-3)=1$. Therefore, if we assume $d<-4,-d$ a prime, $-d \equiv 3(\bmod 4), k=12$ or $k=24$, then we can apply (4). With the fundamental unit $2+\sqrt{3}$ of $\mathbf{Q}(\sqrt{12})$ and $5+2 \sqrt{6}$ of $\mathbf{Q}(\sqrt{24})$ we get the linear form to handle (see, e.g., Stark [15]): If $d<-10^{4},-d$ a prime, $-d \equiv 3$ $(\bmod 4), h(d)=1$, then

$$
\begin{gathered}
h(12 d)<2 \sqrt{|d|}, \quad h(24 d)<2 \sqrt{|d|} \text { and } \\
|h(24 d) \ln (5+2 \sqrt{6})-2 h(12 d) \ln (2+\sqrt{3})|<50 e^{-\pi \sqrt{|d|} / 24} .
\end{gathered}
$$

2. An Upper Bound of Mignotte and Waldschmidt for the Absolute Value of the Discriminant of an Imaginary Quadratic Field. Mignotte and Waldschmidt [11] proved the following theorem: Let $\beta, \alpha_{1}, \alpha_{2}$ denote three nonzero algebraic numbers of exact degrees $D_{0}, D_{1}, D_{2}$, respectively. Let $D$ be the degree over $\mathbf{Q}$ of the field $\mathbf{Q}\left(\beta, \alpha_{1}, \alpha_{2}\right)$. For $j=1,2$ let $\ln \alpha_{j}$ be any determination of the logarithm of $\alpha_{j}$ and let $A_{j}$ be an upper bound for the height of $\alpha_{j}$ and for $\exp \left(\| \ln \alpha_{j} \mid\right)$; put $S_{j}=D_{j}+\ln A_{j}$. Let $B$ be an upper bound for the height of $\beta$ and for $e^{D_{0}}$ and let $S_{0}=D_{0}+\ln B$. Put

$$
T=4+\frac{S_{0}}{D_{0}}+\ln \left(D^{2} \frac{S_{1} S_{2}}{D_{1} D_{2}}\right) .
$$

Then $\Lambda=\beta \ln \alpha_{1}-\ln \alpha_{2}$ satisfies $\Lambda=0$ or

$$
|\Lambda|>\exp \left(-5 * 10^{8} D^{4} \frac{S_{1} S_{2}}{D_{1} D_{2}} T^{2}\right) .
$$

We apply this theorem to the linear form

$$
\Lambda=p \ln (5+2 \sqrt{6})-2 q \ln (2+\sqrt{3}),
$$

where $p$ and $q$ are integers bounded in absolute value by $2 \sqrt{|d|}$. We put $\beta=p / q$, $\alpha_{1}=5+2 \sqrt{6}, \alpha_{2}=2+\sqrt{3}$ and get $D_{0}=1, D_{1}=2, D_{2}=4, D=4, A_{1}=10$, $A_{2}=4, S_{1}=4.3, S_{2}=3.4$; we find the estimation

$$
|\Lambda|>\exp \left(-10^{12} \ln ^{2}|d|\right) \text {. }
$$

Remark. With an adaption of the proof of the theorem of Mignotte and Waldschmidt to our concrete situation, Cherubini [3] gets the better bound

$$
|\Lambda|>\exp \left(-4.6 * 10^{7} \ln ^{2}|d|\right) .
$$

With this estimation one can prove

Proposition 1. If $d \leqslant-10^{4}$ is the discriminant of an imaginary quadratic field with $h(d)=1$, then $d>-10^{34}$.

Proof. From (5) and (7) we derive

$$
\begin{aligned}
25 \exp \left(-\pi \frac{\sqrt{|d|}}{24}\right) & >\left|\frac{h(24 d)}{2 h(12 d)} \ln (5+2 \sqrt{6})-\ln (2+\sqrt{3})\right| \\
& >\exp \left(-10^{12} \ln ^{2}|d|\right)
\end{aligned}
$$


or

$$
\frac{\sqrt{|d|}}{\ln ^{2}|d|}<\frac{24}{\pi}\left(10^{12}+\frac{2 \ln 5}{\ln ^{2}|d|}\right)
$$

For this to hold, it is necessary that $|d|<10^{34}$.

3. Investigation of the Medium Large Region $-10^{34}<d<-10^{4}$ with the Aid of Continued Fractions. Under the assumption of (5), we find a rational approximation to $\gamma=\ln (5+2 \sqrt{6}) / \ln (2+\sqrt{3})$ of the form

$$
\left|\gamma-\frac{2 p}{q}\right|<\frac{50}{\ln (2+\sqrt{3})} \frac{1}{q} \exp \left(\frac{-\pi}{24} \sqrt{|d|}\right), \quad p, q<2 \sqrt{|d|} .
$$

If $h_{n} / k_{n}$ is the $n$th convergent of the continued fraction of $\gamma$, and if $q<k_{n}$, the theory of continued fractions tells us that

$$
\frac{1}{k_{n}\left(k_{n+1}+k_{n}\right)}<\left|\gamma-\frac{h_{n}}{k_{n}}\right|<\left|\gamma-\frac{2 p}{q}\right|
$$

\begin{tabular}{|c|c|c|c|c|c|}
\hline$n$ & $a_{n}$ & $k_{n}$ & $n$ & $a_{n}$ & $k_{n}$ \\
\hline () & 1 & 1 & 1 & 1 & 1 \\
\hline 2 & 2 & 3 & 3 & 1 & 4 \\
\hline 4 & 5 & 23 & 5 & 1 & 27 \\
\hline 6 & 34 & 941 & 7 & 1 & 968 \\
\hline 8 & 5 & 5781 & 9 & 1 & 6749 \\
\hline 10) & 4 & 32777 & 11 & 1 & 39526 \\
\hline 12 & 2 & 111829 & 13 & 5 & 598671 \\
\hline 14 & 3 & 1907842 & 15 & 9 & 17769249 \\
\hline 16 & 46 & 819293296 & 17 & 1 & 837062545 \\
\hline 18 & 9 & 8352856201 & 19 & 163 & 1362352623308 \\
\hline 20) & 2 & 2733058102817 & 21 & 1 & 4095410726125 \\
\hline 22 & 1 & 6828468828942 & 23 & 1 & 10923879555067 \\
\hline 24 & 8 & 94219505269478 & 25 & 8 & 764679921710891 \\
\hline 26 & 3 & 2388259270402151 & 27 & 2 & 5541198462515193 \\
\hline 28 & 6 & 35635450045493309 & 29 & 2 & 76812098553501811 \\
\hline 30 & 1 & 11244754898995120 & 31 & 3 & 414154744350487171 \\
\hline 32 & 9 & 3839840247753379659 & & & \\
\hline
\end{tabular}

must hold. Cherubini calculated the beginning of the continued fraction development of $\gamma$ with a Commodore 64 and found

Since $q<2 \sqrt{|d|} \leqslant 2 * 10^{17}<k_{31}$, we find in combining (8) and (9) that

$$
\frac{1}{k_{31}\left(k_{32}+k_{31}\right)}<38 \exp \left(-\frac{\pi}{24} \sqrt{|d|}\right)
$$

or

$$
\sqrt{|d|}<\frac{24}{\pi}\left(\ln 38+\ln k_{31}+\ln \left(k_{32}+k_{31}\right)\right)<7 * 10^{2}
$$

or $q<1400$. If we look once more in our table, we find that even $q<k_{8}$ must hold, and a further reduction with (10) yields $q<340<k_{6}$. Finally, we find in this way $\sqrt{|d|}<138$. So we have

Proposition 2. If $d \leqslant-10000$ is the discriminant of any imaginary quadratic field with class number one, then $-19044<d \leqslant-10000$. 
4. All Imaginary Quadratic Fields with Discriminant $d$, Class Number One and $d>-19044$. Dickson [4] proves the following lemma.

LEMMA 1. Let $d<-3, d \neq-4,-8$ be the discriminant of an imaginary quadratic field with class number one and let $g=\max \left(n \in \mathbf{N}_{0} \mid 2 n+1 \leqslant(|d| / 3)^{0.5}\right), T_{0}=$ $\frac{1}{4}(|d|+1), T_{j}=T_{0}+j(j+1)$. Then all the numbers $T_{j}, 0 \leqslant j \leqslant g$, are prime.

With the aid of this lemma it is easy to calculate by hand that within $0>d>$ -19044 only the numbers

$$
d=-3,-4,-7,-8,-11,-19,-43,-67,-163
$$

can be discriminants of imaginary quadratic fields with class number one. As is known, Gauss has shown that these numbers have the property in question.

Mathematisches Institut

Albert-Ludwigs-Universität

Albertstrasse 23b

D-7800) Freiburg. West Germany

1. A. BAKғR, "Linear forms in the logarithms of algebraic numbers," Mathematica, v. 13, 1966, pp. 2()4-216.

2. P. BundsChuH \& A. HocK, "Bestimmung aller imaginär-quadratischen Zahlkörper der Klassenzahl Eins mit Hilfe eines Satzes von Baker," Math. Z., v. 111, 1969, pp. 191-204.

3. J. Chirubini, Ein neuer Beweis des Stark-Heegnerschen Satzes, Diplomarbeit, Freiburg, 1985.

4. L. E. DickSON, "On the negative discriminants for which there is a single class of positive primitive binary quadratic forms," Bull. Amer. Math. Soc., v. 17, 1919, pp. 534-537.

5. N. I. Fel'dman \& N. G. Chudakov, “On Stark's theorem,” Math. Notes, v. 11, 1972, pp. 204-210.

6. C. F. Gauss, Untersuchungen über höhere Arithmetik, Göttingen, 1889 (Neuauflage, Chelsea, 1965).

7. A. O. (BEL'FOND), "Approximating algebraic numbers by ratios of logarithms of two algebraic numbers," Izl. Akad. Nauk SSSR Ser. Mat., Nos. 5-6, 1939, pp. 509-518. (Russian)

8. B. H. Gross \& D. B. ZAGIER, "Heegner points and derivatives of $L$-series," Invent. Math., v. 84, 1986, pp. 225-320.

9. K. Heigniz, "Diophantische Analysis und Modulfunktionen,” Math. Z., v. 56, 1952, pp. 227-253.

10. H. HIILBRONN \& E. H. LinfOOT, "On the imaginary quadratic corpora of class-number one," Quart. J. Math. Oxford Ser., v. 5, 1934, pp. 293-301.

11. M. MignotTE \& M. Waldoschmidt, "Linear forms in two logarithms and Schneider's method," Math. Ann., v. 231, 1978, pp. 241-267.

12. H. Stark, "On complex quadratic fields with class number equal to one," Trans. Amer. Math. Soc., v. 122, 1966, pp. 112-119.

13. H. M. Stark, "On the "gap" in a theorem of Heegner," J. Number Theory, v. 1, 1969, pp. 16-27.

14. H. M. STARK, "A complete determination of the complex quadratic fields of class-number one," Michigan Math. J., v. 14, 1967, pp. 1-27.

15. H. M. Stark, On the Problem of Unique Factorization in Complex Quadratic Fields, Number Theory. Proc. Sympos. Pure Math., vol. 12. Amer. Math. Soc., Providence, R. I., 1969. pp. 41-56. 